\title{
Differential regulation of two closely related integrative and conjugative elements from Streptococcus thermophilus
}

Nicolas Carraro ${ }^{1,2}$, Virginie Libante ${ }^{1,2^{*}}$, Catherine Morel $^{1,2}$, Bernard Decaris ${ }^{1,2}$, Florence Charron-Bourgoin ${ }^{1,2}$, Pierre Leblond ${ }^{1,2}$ and Gérard Guédon ${ }^{1,2}$

\begin{abstract}
Background: Two closely related ICES, ICESt1 and ICESt3, have been identified in the lactic acid bacterium Streptococcus thermophilus. While their conjugation and recombination modules are almost identical (95\% nucleotide identity) and their regulation modules related, previous work has demonstrated that transconjugants carrying ICESt3 were generated at rate exceeding by a 1000 factor that of ICESt1.

Results: The functional regulation of ICESt1 and ICESt3 transcription, excision and replication were investigated under different conditions (exponential growth or stationary phase, DNA damage by exposition to mitomycin C). Analysis revealed an identical transcriptional organization of their recombination and conjugation modules (long unique transcript) whereas the transcriptional organization of their regulation modules were found to be different (two operons in ICESt1 but only one in ICESt3) and to depend on the conditions (promoter specific of stationary phase in ICESt3). For both elements, stationary phase and DNA damage lead to the rise of transcript levels of the conjugation-recombination and regulation modules. Whatever the growth culture conditions, excision of ICESt1 was found to be lower than that of ICESt3, which is consistent with weaker transfer frequencies. Furthermore, for both elements, excision increases in stationary phase (8.9-fold for ICESt1 and 1.31-fold for ICESt3) and is strongly enhanced by DNA damage (38-fold for ICESt1 and 18-fold for ICESt3). Although ICEs are generally not described as replicative elements, the copy number of ICESt3 exhibited a sharp increase (9.6-fold) after mitomycin C exposure of its harboring strain CNRZ385. This result was not observed when ICESt3 was introduced in a strain deriving ICESt1 host strain CNRZ368, deleted for this element. This finding suggests an impact of the host cell on ICE behavior.

Conclusions: All together, these results suggest a novel mechanism of regulation shared by ICESt1, ICESt3 and closely related ICEs, which we identified by analysis of recently sequenced genomes of firmicutes. This is the first report of a partial shutdown of the activity of an ICE executed by a strain belonging to its primary host species. The sharp increase of ICESt3 copy number suggests an induction of replication; such conditional intracellular replication may be common among ICEs.
\end{abstract}

\section{Background}

Acquisition of genomic islands (GIs) plays a key role in bacterial evolution $[1,2]$. In silico analyses revealed that numerous GIs probably belong to Integrative and Conjugative Elements (ICEs) or are ICE-deriving elements $[3,4]$. ICEs, including conjugative transposons, were defined as autonomous mobile elements that encode the

\footnotetext{
* Correspondence: Virginie.Libante@scbiol.uhp-nancy.fr

'Nancy-Université, UMR1128, Génétique et Microbiologie, F-54506

Vandœuvre-lès-Nancy, France

Full list of author information is available at the end of the article
}

functions needed for their excision, conjugative transfer and integration [3].

Cis-acting sequences and genes involved in a same biological process (for example conjugation) are generally grouped in a module, such as oriT and genes encoding relaxosome and conjugation pore. The recombination, conjugation and regulation modules are frequently grouped to form the core region of the ICEs. Although ICEs replicate during their conjugative transfer, it was originally assumed that they are incapable of autonomous intracellular replication and that their
C Biomed Central 
maintenance during cell growth and division only relies on their integration in the chromosome. Besides one or few core regions, they also harbor highly variable regions that encode functions potentially useful for the bacterial host [5]. Comparison of the organization of related ICEs, such as $\operatorname{Tn} 916$ and its close relatives, revealed that they evolve by deletion, acquisition and/or exchange of modules. The conjugation, tetracycline resistance and regulation modules of Tn916 and Tn5397 are closely related whereas their recombination modules are unrelated [6]. Likewise, the Tn1549 recombination module is closely related to the one of Tn916, but their conjugation and resistance modules are unrelated [7].

The closely related ICEs of the lactic acid bacterium Streptococcus thermophilus, ICESt1 and ICESt3, are integrated within the 3' end of the $f d a$ gene encoding a putative fructose 1,6-diphosphate aldolase [8,9]. They carry recombination and conjugation modules that are almost identical (95\% nucleotide identity), related regulation modules (three homologous genes showing about $85 \%$ identity; to two or three unrelated genes) and various modules that could be advantageous for their hosts (including phage resistance). Their conjugation modules are very distantly related to modules of a large group of ICEs found in firmicutes, including Tn916 and ICEBs1 [8]. As the conjugative transfer of ICESt1 occurs at a frequency one thousand times lower than that of ICESt3, their divergent regulation modules might be involved in these very different transfer activities [10].

The activity of almost all prophages and at least some ICEs is controlled by a central repressor that can belong to two unrelated families, either cI or ImmR (also known as cI-like, although they are not homologous to cI repressor). Both types of repressor carry a HTH XRE domain that allows their binding to promoter sequences upstream from their target genes. Transfer of the element requires the inactivation of the corresponding regulator, as shown during the RecA-dependent SOS response [11-13] of many cI-encoding prophages and two ICEs, SXT from Vibrio cholerae [14] and ICEBs1 from Bacillus subtilis [12], which encode respectively a CI and an ImmR repressor. Derepression of the ICE is due to the cleavage of the transcriptional regulator catalyzed by either the cI autopeptidase function [15] or a metalloprotease encoded by a gene adjacent to the gene encoding ImmR $[12,16]$. Previous studies showed that various stimuli can activate ICEs, such as antibiotic treatment, cell density, stationary phase, DNA damage or presence of chlorocatechol $[5,11,15]$.

Within the regulation module of ICESt1 and ICESt3, genes encoding homologs of cI (named arp1) and $\operatorname{ImmR}(\operatorname{arp} 2)$ and its associated protease (orfQ) were identified. ICESt1 and ICESt3 are the only two characterized elements which encode both $\mathrm{cI}$ and ImmR repressors, suggesting a novel and complex regulatory mechanism.

In order to explain the differences of transfer frequency previously observed for ICESt1 and ICESt3 of S. thermophilus, a transcriptional mapping of these elements was undertaken. Furthermore their excision/replication rates were investigated in different conditions (growth medium, exponential growth, stationary phase, after exposure to DNA damaging agent). Finally the influence of the host background was also explored. These experiments revealed that the two ICEs harbor closely related core regions, differ in their transcriptional organization and regulation. They provide further evidence of ICE replication. Our results also pointed out an impact of host cell on the ICE behavior.

\section{Results}

Transcriptional organization and promoter analyses of the ICESt 1 and ICESt3 core region

Previous sequences analyses suggested that the thirteen ORFs belonging to the conjugation module and the genes encoding the excisionase and integrase (recombination module) of ICESt1/3 could be transcribed as a unique polycistronic mRNA while the regulation module could have a two-operon organization [11]. Gene organization, position of predicted promoters and rho-independent transcription terminators of the ICESt1/3 core region are schematically presented in the Figure 1 . As some ICE activities were reported to be affected by growth phase and/or cell density $[17,18]$, CNRZ368 and CNRZ385, strains carrying ICESt1 and ICESt3 respectively, were harvested in exponential growth phase as well as in stationary phase for total RNA extraction and subsequent transcriptional organization studies.

To determine which genes were co-transcribed, RTPCR amplification of core region was performed by grouping ORFs two by two or three by three. For ICESt1, amplifications of orfR/arp1/orfQ and orfP/arp2, respectively, were positive while that of the orfQ/orfP junction was negative (see additional file 1: S1B). These data comfort the hypothesis of a two-operon organization for ICESt1 (see additional file 1: S1A) with a functional rho-independent transcription terminator located between the two operons. By contrast, for ICESt3, all the RT-PCR amplifications of the regulation module were positive (see additional file 1: S1D) indicating a cotranscription of all the regulation genes (see additional file 1: S1C). The free energy of the transcriptional terminator detected between orf385B and orfQ genes in ICESt3 (Figure 1) was calculated with the mFold software [19]. It is different from the one for ICESt1 $(\Delta G=$

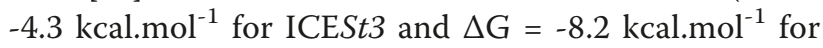
ICESt1). This difference could explain why all genes of the regulation module of ICESt3 can be co-transcribed 
while two independent transcriptional units were found in ICESt1.

We then examined the activity of the promoter located upstream from the orfQ gene by Rapid Amplification of cDNA ends (5' RACE). For both elements, the start point
(A nucleotide) was located seven nucleotides downstream from a -10 box separated by 17 nt from a -35 box, which overlapped the rho-independent transcription terminator (Figure 1A). This result is consistent with the S. thermophilus promoter consensus sequence (TTGACA - 17 nt - 
TATAAT) [20]. Therefore, both ICEs possess a functional $\mathrm{P}_{\text {orfo }}$ promoter. However, it was previously showed that ICESt3 differs from ICESt 1 by a -1 frameshift in the 5 ' end of its orfQ gene (orfQ1) [11]. A second RBS, that could enable the translation from an initiation codon located downstream, was identified in silico (Figure 1A). All together, these data suggest that the orfQ2 gene of ICESt3 is truncated of 54 nucleotides at its 5' end compared to the orfQ gene of ICESt1.

All RT-PCR amplifications targeting co-transcription of the sixteen conjugation-recombination genes of ICESt1 and ICESt3 gave amplicons (see additional file 1: S1B and S1D). Therefore, these genes are transcribed as a single polycistronic mRNA of about $14.6 \mathrm{~kb}$ (see additional file 1: S1A and S1C). To map more precisely the 5' end of these transcripts, other sets of primers were designed in the $\operatorname{arp} 2 / \operatorname{orf} N$ intergenic region. For ICESt 1 , these results (data not shown) combined with 5' RACE experiments confirmed the predicted conjugation-recombination promoter, $\mathrm{P}_{\mathrm{cr}}$, with a -10 box (TATAAT) located seven nucleotides upstream from the transcription start point (A) nucleotide (Figure 1B). RT-PCR experiments also localized the ICESt3 $\mathrm{P}_{\text {cr }}$ promoter in the same region, between the $f 4$ and $f 3$ primers (Figure $2 \mathrm{~A}$ and $2 \mathrm{~B}$ ). The ICESt3 precise start point could not be deduced from 5'RACE experiments because all the obtained products ended in a region located 100 bp downstream from the corresponding start point of ICESt1. For ICESt1, several 5'RACE products also ended in this region. mFold software analysis [19] revealed a conserved putative stem loop structure $(\Delta \mathrm{G}=-6.7 \mathrm{kcal}$. $\mathrm{mol}^{-1}$ for ICESt 1 and $\Delta \mathrm{G}=-6.4 \mathrm{kcal} \cdot \mathrm{mol}^{-1}$ for ICESt3), which could affect RNA stability. Although it could not be experimentally demonstrated, we propose, based on sequence conservation (Figure 1B), a same location of the $\mathrm{P}_{\text {cr }}$ promoter for ICESt3 and ICESt1.

For both elements, the functionality of the predicted arp2 promoter $\mathrm{P}_{\mathrm{arp} 2}$ was established with a (A) start site located seven nucleotides downstream from a -10 box (TACAAT) (Figure 1B). For both ICEs, transcriptional analyses showed that all the promoters $\left(\mathrm{P}_{\mathrm{cr}}, \mathrm{P}_{\mathrm{orfQ}}\right.$ and $\mathrm{P}_{\text {arp2 }}$ ), which are active during the stationary phase, are also active during exponential the growth phase (data not shown). However, an additional promoter was identified in ICESt3 upstream from the $\mathrm{P}_{\text {arp2 } 2}$ promoter during stationary phase. Amplicons were obtained using arp2.f/r3 and arp2.f/r4 primers (Figure 2C). 5'RACE experiments revealed a start site located within a $(\mathrm{A})_{6}$ stretch in this region (between the $r 4$ and $r 5$ primers, Figure 2C). Therefore, an alternative transcript originating from a distal arp 2 promoter in ICESt3 (called " $\mathrm{P}_{\text {arp2s }}$ ") is expressed during the stationary phase (Figure $1 \mathrm{C}$ ). This promoter does not match the classical promoter consensus as its -35 (TTATCA) and -10 (TGTAAT) boxes are separated by only 15 nucleotides (Figure 1C). The functionality of this

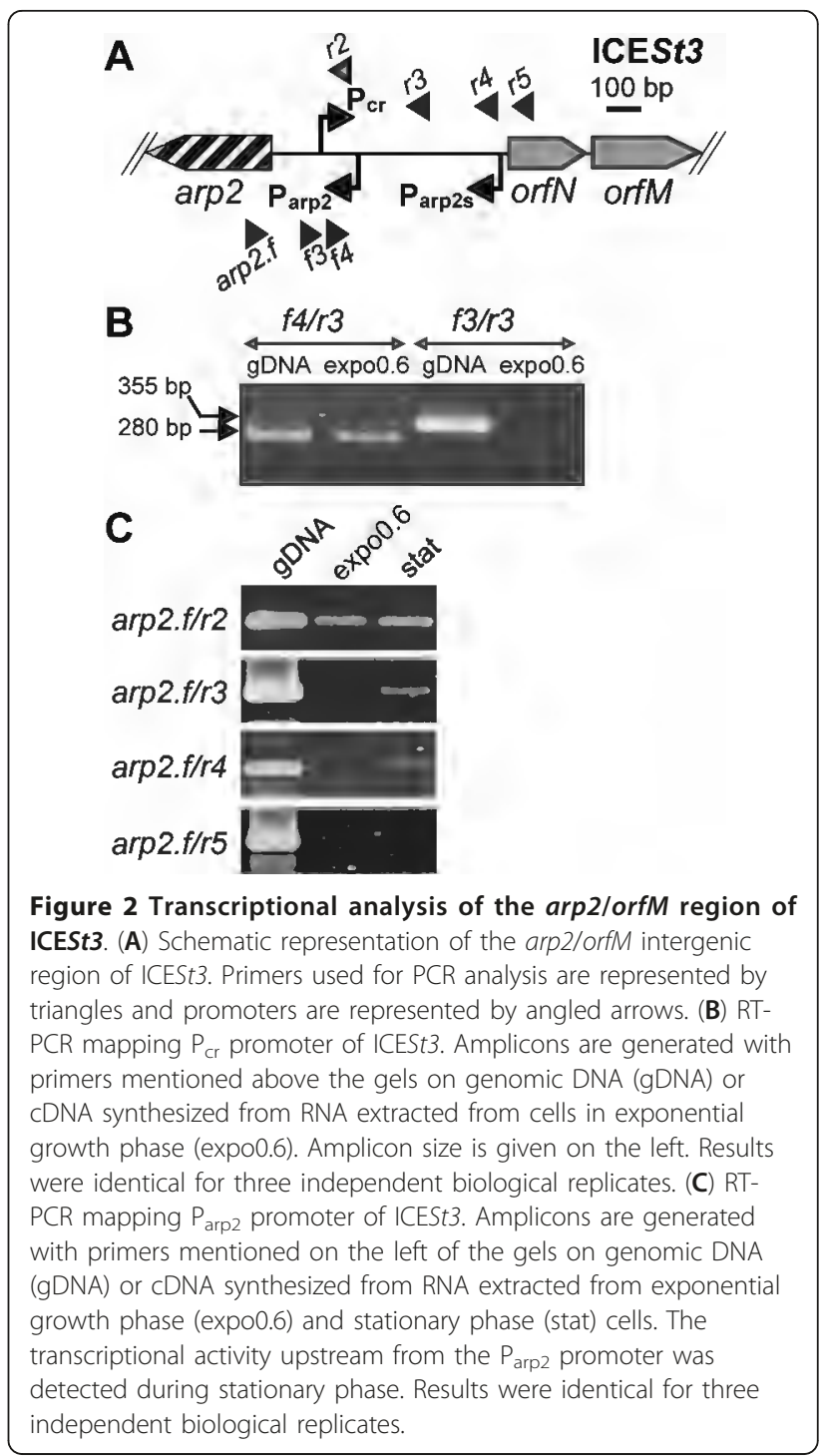

promoter was highlighted only during stationary phase (Figure 2C) and only in ICESt3 (data not shown), although its sequence is strictly identical in ICESt1 (Figure 1C). Sequence analyses failed to detect any ORF in the 389 nucleotides between the $\mathrm{P}_{\text {arp2s }}$ and $\mathrm{P}_{\text {arp2 } 2 \text { promoters. }}$

Taken together, these data demonstrate that ICESt1 and ICESt3 do not share the same transcriptional organization of their regulation module: ICESt1 is organized as two operons, while in ICESt 3 the whole module can be co-transcribed. Furthermore, ICESt3 possesses an additional distal promoter upstream the module, which is activated during stationary phase.

\section{Growth phase and MMC exposure modulate the transcription of the ICESt 1 and ICESt3 core genes} Previous analyses showed a derepression of conjugative transfer of ICESt 3 but not of ICESt1 after exposure to 
mitomycin C (MMC) [10]. In order to explain this difference, we quantified by real-time RT-PCR, three regions (orfM/orfL junction, orfD/orfC junction and integrase gene) of the conjugation-recombination transcript of ICESt1 and ICESt3.

Quantification was done from cells harvested in exponential growth phase treated or not with $\mathrm{MMC}$ at the half of the minimal inhibitory concentration $(\mathrm{MIC} / 2)$ as well as in stationary phase (Figure 3). Of note, in preliminary experiments, MMC exposure did not affect the transcriptional organization (in particular no activity of ICESt3 $\mathrm{P}_{\text {arp2s }}$ ), cell morphology or chain length but, as expected for a DNA damaging agent, it delayed growth, reduced DNA quantity and increased $\operatorname{rec} A$ transcript levels (data not shown). Transcription of the ICESt1 conjugation-recombination modules was found up-regulated upon DNA damage (16-fold for the int gene) and in stationary phase (13-fold for the int gene) compared to exponential growth phase without MMC treatment (Figure 3A). The same observation was made for ICESt3 with a 84 -fold and 11-fold increase of int transcript levels after MMC treatment and stationary phase, respectively (Figure 3B), indicating a probable transcriptional regulation of ICE excision. Whatever the considered region of the conjugation-recombination transcript, higher amounts were found for ICESt3 than for ICESt1 (for example, 16 to 100-fold difference in int gene transcript level depending on the tested condition).

For both elements, quantitative RT-PCR was also performed on three loci of the regulation module (Figure $3)$. In ICESt1, the amount of arp2-orfP transcripts was similar whatever the conditions considered, while the amount of arp1 transcripts increased 10-fold after MMC treatment (Figure 3A). Regardless of conditions, no amplification was detected at the junction between the two operons (orfQ/orfP junction), which corroborates the lack of cotranscription of these genes. For ICESt3, the level of arp1 and orf385A/arp 2 transcripts increased after MMC treatment (40-fold) and in stationary phase (about 10-fold) (Figure 3B). Co-transcription of the two operons was quantified by considering the orfQ/orf385B junction. During exponential growth phase and MMC exposure, co-transcription represented 20 and $38 \%$ of transcripts respectively, indicating that the




terminator and the promoter $\mathrm{P}_{\text {orfQ }}$ were active. However, in stationary phase, the amount of this junction was similar to that of the two operons, probably reflecting an activity of the $\mathrm{P}_{\text {arp2s }}$ promoter.

After MMC exposure during stationary phase, transcript quantities were found to be similar to the ones observed in stationary phase without MMC. Therefore, MMC has an impact on DNA metabolism (lower level of DNA) during stationary phase but does not affect levels or organization of transcripts (data not shown).

\section{Growth phase and mitomycin C affect ICESt1 and ICESt3 excision}

Excision is the first step of ICE transfer from host chromosome to a recipient cell, leading to a circular intermediate and an empty chromosomal integration site, attB (Figure 4A). The influence of the growth phase (early, mid exponential growth phase or stationary phase) and MMC treatment on ICE excision was analyzed by quantitative PCR on genomic DNA. The excision percentage was calculated as the copy number of at $t B$ sites per $f d a$ copy (adjacent chromosomal locus). As a control, the amount of $a t t B$ sites was determined in strain CNRZ368 $\triangle$ ICESt1 (X. Bellanger unpublished data) and in CNRZ385 IICESt3 [21] and was found equal to the amount of $f d a$.

The excision percentage of ICESt3 was found sevenfold higher than the one of ICESt1 in exponential growth phase (Figure 4B), consistent with the higher level of ICESt3 conjugation-recombination transcript (described above), and its higher transfer frequency [10]. For both ICEs, excision frequency was higher in stationary phase compared to exponential growth phase (Figure $4 \mathrm{~B})$. For these experiments, cells were grown in LM17 rich medium, in which transfer has been demonstrated [10]. A similar excision rate of ICESt3 was measured in another rich medium (HJGL medium) that do not support the transfer of the two ICEs (data not shown). Therefore, the lack of ICESt 3 transfer in this medium can not be due to a low excision level.

Transcriptional analyses have shown an increase of core transcript level for ICESt3 and ICESt1 after MMC treatment during exponential growth. This DNA damaging agent leads to an increase of excision percentage up to $90 \%$ for ICESt3, but only $4.3 \%$ for ICESt1 (Figure 4C). However, the increase is higher for ICESt1 (38fold) compare to ICESt3 (18-fold). Therefore, under all tested conditions, ICESt 3 is more active in excision than ICESt1.

\section{DNA damage induces replication of ICESt3}

Quantitative PCR was performed to measure the amounts of excised and integrated ICEs at different growth phases and after MMC treatment. According to

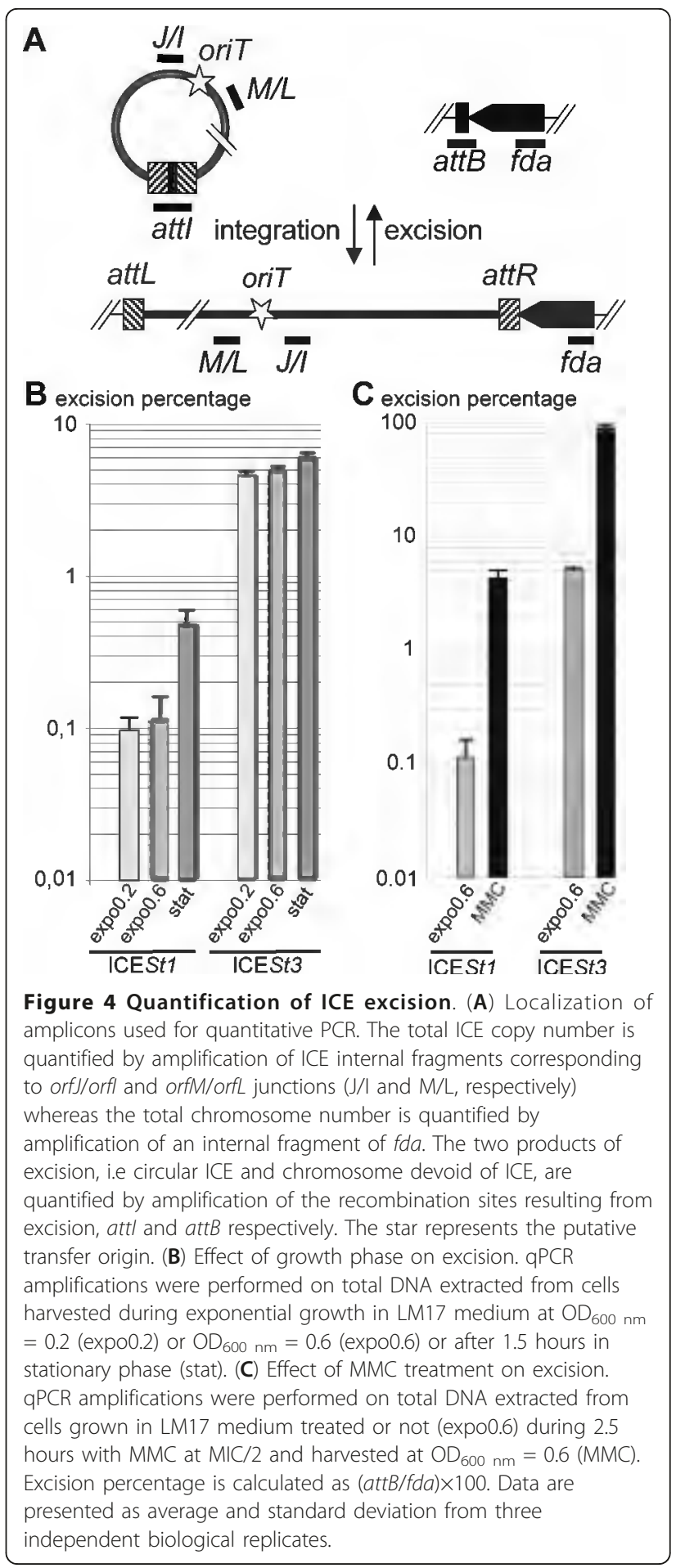

the previously proposed ICE model (Figure 4A) attI and $a t t B$ were expected to have the same copy number after ICE excision. This was found for both ICEs whatever the tested conditions, except for ICESt3 DNA extracted from strain CNRZ385 exposed to MMC (with a attI/ $a t t B$ value of $9.95 \pm 1.42$ ). To confirm this data, the 
orfM/orfL junction localized in the conjugation module was quantified and normalized to levels of different chromosomal loci: $f d a, d n a A$ and $x e r S$ (data not shown). The same result was obtained with an amount of $M / L$ reaching about nine-fold the one of $f d a(9.60 \pm 1.04)$. As $f d a$ is adjacent to integrated ICESt 3 and replicates prior to the ICE during host chromosome replication, ICESt3 could be able to replicate autonomously under this condition. Different loci along ICEs (from $J / I$ to $M$ / $L$ ) were quantified at similar levels (data not shown) and thus did not allow us to propose a replicative mechanism (theta $v / s$ rolling-circle).

ICESt3 excision and replication depend on the host strain To test the ICESt3 behavior in different $S$. thermophilus strain background, its excision percentage (attB/ $f d a) \times 100$ and copy number $(M L / f d a)$ were quantified. ICESt3 was transferred by conjugation to LMG18311, a strain initially devoid of ICE and in CNRZ368 $\mathrm{ICESt1}$, the strain that originally carries ICESt1 but has been deleted of it. ICESt3 excision percentage was lower in strain LMG18311 and much lower in CNRZ368 $\Delta$ ICESt1 compared to that observed in CNRZ385 strain but MMC treatment increased its excision percentage in all strains (Figure 5). In CNRZ368, excision rates of ICESt3 were higher than those of ICESt1 (Figure 5). Furthermore, the quantification showed a single copy of ICESt3 (1.08 \pm 0.11$)$ per chromosome even after MMC exposure (compared to $9.60 \pm 1.04$ copies in strain CNRZ385). This indicates a preponderant effect of the host strain on the ICE replication.

\section{A family of streptococcal ICEs shares related regulation and conjugation modules}

Protein and nucleic acid sequences from the regulation, conjugation and recombination modules of ICESt1 and ICESt3 were compared with sequences from firmicutes. Closely related conjugation modules (> 80\% nucleotide identity all along the conjugation module) were found in the putative ICESpn8140 from S. pneumoniae 8140 [22] and in the partially or completely sequenced genomes of S. parasanguinis ATCC15912 and F0405, S. infantis ATCC 700779 and S. australis ATCC700641 (Figure 6). All these conjugation modules are adjacent to putative recombination modules that are unrelated or very distantly related to the ones of ICESt1/3 (data not shown). Nevertheless, they could be cotranscribed with the conjugation module from a $\mathrm{P}_{\mathrm{cr}}$ promoter similar to the one identified above since it is present at the same position as in ICESt1/3 with high sequence conservation (see additional file 2: S2A). Therefore, these conjugation-recombination modules probably belong to non identified ICEs.

All these putative elements harbor closely related regulation modules that would be transcribed divergently



Figure 5 Strain effect on ICE excision. qPCR amplification was performed on total DNA extracted from cells harvested during exponential growth in LM17 medium at $\mathrm{OD}_{600 \mathrm{~nm}}=0.6$ (expo0.6) or treated for 2.5 hours by MMC at MIC/2 and harvested at $\mathrm{OD}_{600} \mathrm{~nm}=$ 0.6 (MMC). ICE and host strains studied are indicated below. ICESt3, in strains CNRZ368 and LMG18311, was tagged with the cat gene, conferring chloramphenicol resistance, for transconjugant selection. To avoid ICE interference, strain CNRZ368 was previously deleted of ICESt1 prior ICESt3cat transfer. Excision percentage is calculated as $(a t t B / f d a) \times 100$. Data are presented as average and standard deviation from three independent replicates.

from the conjugation and recombination modules. Al these modules possess a similar organization and encode putative cI repressors, ImmR repressors and metalloproteases related to the ones of ICESt1/3 (64-90\% protein sequence identity) and one to four unrelated proteins (Figure 6). Sequence comparison of the intergenic core regions of the closely related streptococci ICEs revealed similar regulatory signals at the same positions as in ICESt1/3 with high sequence conservation (see additional file 2: S2B, S2C and S2D), suggesting a similar regulation.

More distantly related conjugation modules (35-70\% identity for at least seven proteins with similar organization) are found not only in previously described elements - RD2 from S. pyogenes [23] and four elements integrated in a tRNA ${ }^{\text {Lys }}$ gene from four S. agalactiae strains [4] - but also in novel putative ICEs that we found in various Streptococci including S. agalactiae ATCC13813 (incompletely sequenced), S. dysgalactiae ATCC12394 (two elements), S. downei F0415, Streptococcus sp. 2_1_36FAA and S. gallolyticus UCN34. Only the elements found in $S$. dysgalactiae encode a putative cI repressor, ImmR repressor and metalloprotease. 


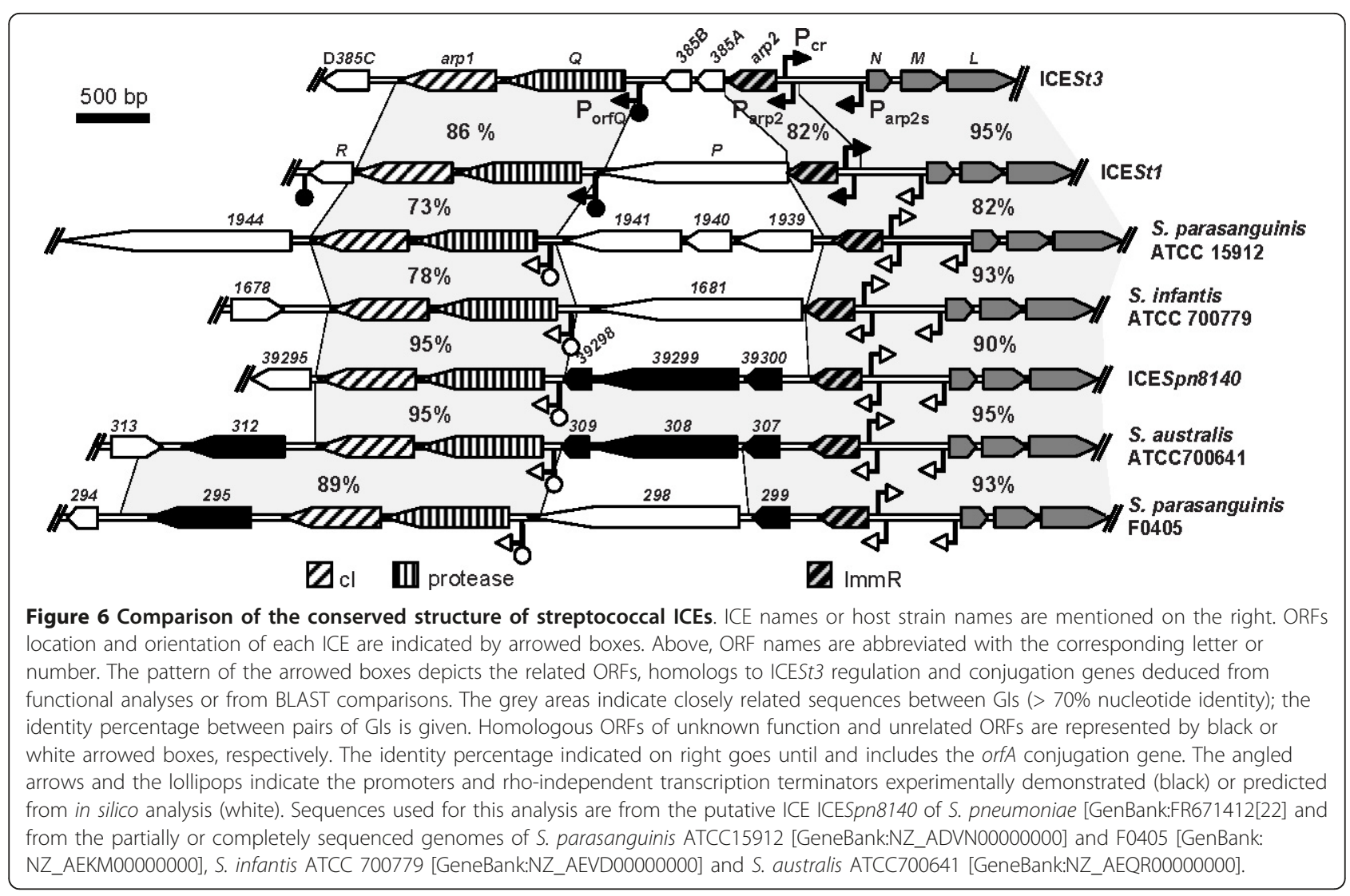

\section{Discussion}

This study of ICESt1 and ICESt3, showed that their respective transcriptional organization and their mobility behaviors differ. As previously proposed from sequence analyses, all genes included in the conjugation and recombination modules of the two elements were found to be transcriptionally linked and controlled by a single promoter. This organization allows a coordinated regulation of genes involved in conjugation and recombination, which are functionally associated during ICE transfer.

For ICESt1 and ICESt3 regulation module, the cI-like encoding gene and one to two genes located downstream are expressed from the convergent promoter $\mathrm{P}_{\mathrm{arp} 2}$ or from a distal conditional promoter $\mathrm{P}_{\mathrm{arp} 2 \mathrm{~s}}$. The genes encoding metalloprotease (orfQ) and cI homologs belong to a different operon expressed from another promoter $\mathrm{P}_{\text {orfQ }}$. These two operons are separated by a rho-independent transcription terminator. The ICESt1 regulation module includes two independent transcriptional units. By contrast, co-transcription of all the ORFs belonging to the regulation module was observed for ICESt3. This is probably enabled by a weaker transcriptional terminator and perhaps a higher transcription level and the activation of the stationary phase promoter $\mathrm{P}_{\text {arp2s. }}$. These differences probably induce ICESt3 and ICESt1 differential regulations.
The mechanisms of ICE regulation based on $\mathrm{cI}$ or ImmR repressors, previously described for SXT and ICEBs 1, are characterized by a decrease of transcript level of the cI or $i m m R$ gene and an activation of the conjugation-recombination module transcription [5]. By contrast, in ICESt3 from S. thermophilus, a transcriptional derepression was observed for the two operons of the regulation module, whereas in ICESt1, only the transcript level of the operon containing arp1 was affected. Under all tested conditions, ICESt3 is more transcriptionally active than ICESt1. The partial derepression of transcription of the regulation module may explain the lower activation of ICESt1 (conjugation-recombination transcript level, excision, replication) compared to ICESt3. So far, ICESt1 and ICESt3 were the only known elements (ICEs and prophages) encoding homologs of both $\mathrm{cI}$ and ImmR repressors. The gene encoding a putative metalloprotease is generally cotranscribed and located immediately downstream from the gene encoding the ImmR repressor $[12,16]$. However, in ICESt1 and ICESt3, the metalloprotease gene (orfQ) is adjacent to the cI gene (arp1) but not to the cI-like gene (arp2), suggesting that the regulation involving both $\mathrm{cI}$ and cIlike regulators fundamentally differs from those identified in ICEs and related elements encoding only one regulator. Genomic analyses revealed, in various 
streptococci, ICEs that harbor conjugation module related to the ICESt $1 / 3$ ones These elements carry a regulation module related to the ICESt1/3 ones, suggesting that they could share a similar regulation.

After MMC treatment, the transcript levels of the recombination module increases 16-fold for ICESt 1 and 84-fold for ICESt3. The 10-fold increase in ICESt3 copy number, after MMC treatment, could contribute to this increase of transcript levels but is not sufficient to explain its range. MMC exposure could induce an overinitiation of DNA replication with an apparent increase in origin-proximal gene expression for a short distance $(\approx 50 \mathrm{~kb})$ [24], but ICESt1 and ICESt3 are out of this area on the chromosome. MMC thus stimulates ICE transfer $[10,15,25]$, but also increases transcription of both ICESt3 and ICESt1.

As copy number of ICESt3 increases after MMC treatment, the quantification of the empty chromosomal integration site underestimates the level of extrachromosomal ICEs. It is worth noticing that the increase of excision after MMC exposure does not lead to an increase of ICESt1 transfer. Additionally, a similar excision level was obtained for ICESt3 in HJGL medium, although this medium does not support ICE transfer. It shows that, besides excision, additional factors affect transfer of these elements. Similarly, although prior excision is required to observe the conjugative transfer of Tn916, which is an ICE that harbors a conjugation module very distantly related to the one of ICESt1/3, the transfer frequency of this ICE is not correlated with excision [26].

Some preliminary results favor the hypothesis of multiple extrachromosomal copies of ICESt3 (data not shown). ICEs, as their name implies, are able to excise from their host chromosome. Then the circular extrachromosomal ICE transfers to recipient cell per conjugation and simultaneously replicates by rolling-circle mechanism. The site-specific recombination leads to integration in donor and recipient chromosomes. During division, ICE transmission to the daughter cells is thought to depend on the replication and partition of the host chromosome. However, it has been recently reported that at least some ICEs can replicate independently of their conjugative transfer. In particular, the amount of excised forms of ICEBs1 increases two- to five-fold under inducing conditions [27] ICEBs1 replication is initiated within oriT and is unidirectional [27]. This replication is involved in the stability of ICEBs 1 and required the relaxase encoded by the element. In silico analysis of the putative relaxases of ICESt1/3 and of ICEBs 1 indicated that they are distantly related (27.4\% amino acid identity for relaxase), suggesting that replication could have similar role for the two ICEs.
Furthermore, the ICE RD2 from S. pyogenes related to ICESt1/3 [23] and the putative ICE pKLC102 from Pseudomonas aeruginosa [28] were reported to be simultaneously integrated and at extrachromosomal multiple copies while pP36 from Legionella pneumophila is present as a multiple extrachromosomal copies in some conditions [29]. Whereas, in firmicutes, none of the known ICEs was found to encode a partitioning system; in proteobacteria, the ICEs belonging to pKLC102ICEclc family encode a putative partition system [30,31].

In its host strain CNRZ368, ICESt1 exhibits a stable copy number, even after a stimulation of its excision and core region transcription by MMC exposure. In this strain, ICESt3 excision percentage is reduced 3-fold in stationary phase and nine-fold after MMC treatment and ICESt3 copy number is not increased compared to the one observed in the strain CNRZ385. Additional factor(s) could explain these differences (excision percentage and copy number) of ICESt 3 in different $S$. thermophilus strains. Some host factors are likely involved in key steps of the ICE behavior, like B. subtilis PolC, DnaN and PcrA for ICEBs1 replication [27] and IHF for SXT excision in $V$. cholerae [32]. To our knowledge, our work is the first report of partial shutdown of ICE activity by a strain belonging to the primary host species.

Analysis of recently available sequences led us to identify a set of closely related putative ICEs among various streptococcal species. All of them exhibit closely related conjugation modules but highly variable recombination modules. This suggests that these elements can transfer between various streptococcal species and exchange modules between one another. However, these regulation modules all share $\operatorname{arp} 2$, orfQ and arp1 genes (Figure 6), suggesting a fundamental function of these 3 genes in governing transfer of this ICE family. Further investigations will be required to characterize these genes and of their functional interactions with host regulators.

\section{Conclusions}

In conclusion, the transcriptional organization of the conjugation and recombination modules of two closely related ICEs from S. thermophilus, ICESt1 and ICESt3, is identical, while that of their regulation module is somewhat different. Transcripts of core region and excision levels are higher for ICESt3, which is consistent with its higher transfer frequency. Despite these differences, the excision of both ICEs is stimulated by exposure to a DNA damaging agent and stationary phase. Data generated by the transcriptional study suggest a new mechanism of regulation of ICESt1/3. This behavior could be due to the atypical regulation module of these elements that encode homologues of both $\mathrm{cI}$ and ImmR repressors. Analyses of sequenced genomes revealed, 
among streptococci, a family of ICEs that encode cI and ImmR homologs and therefore could share similar regulation.

Furthermore, our results suggest that DNA damage induces not only the excision and transfer of ICESt 3 but also its intracellular replication. This characteristic, which is not considered in the initial ICE model, may be shared by other ICEs. This study also revealed that ICESt3 has very different behaviors depending on its primary host species, suggesting a major role of host factor(s) in its excision and replication.

\section{Methods}

\section{Strains and media}

The Escherichia coli and S. thermophilus strains used are listed (Table 1). E coli DH5a (Gibco Life Technologies, Gaithersburg, Md, USA.) used for plasmid propagation and cloning experiments was routinely grown in LB medium at $37^{\circ} \mathrm{C}$ in aerobiosis [33]. S. thermophilus strains were grown in M17 broth (Oxoid, Dardilly, France) supplemented with $0.5 \%$ lactose (LM17) and $1 \%$ glucose (GLM17) or Hogg-Jago broth [34] supplemented with $1 \%$ glucose and $1 \%$ lactose (HJGL), at $42^{\circ} \mathrm{C}$ under anaerobic conditions (GENbox Anaer atmosphere generators and incubation jars from bioMérieux, Craponne, France). Agar plates were prepared by adding 2\% (wt/ vol) agar to the media.

\section{Strain CNRZ368 ICESt3cat construction}

To test the ICESt3 behavior in different S. thermophilus strain background, a filter mating was done as described previously [10] using the donor strain CNRZ385, carrying ICESt 3 tagged with the cat gene conferring the chloramphenicol resistance [10] and the recipient strain CNRZ368 $\triangle$ ICESt1, spontaneous rifampicin and streptomycin-resistant mutant (X. Bellanger unpublished data).
Triple-resistant clones were isolated and mapped for cse gene polymorphism [35] to confirm that they are transconjugants harboring CNRZ368 ICESt3cat. Three independent CNRZ368 ICESt3cat clones, which have similar growth parameters, mitomycin $\mathrm{C}$ (MMC) minimal inhibitory concentration (MIC) and dnaA/xerS rates (exponential growth phase with and without MMC treatment and stationary phase) than strains CNRZ368 and CNRZ368 cured of ICESt1 were used for each experiments.

\section{Growth conditions}

S. thermophilus strains were grown at $42^{\circ} \mathrm{C}$ in $30 \mathrm{~mL}$ of LM17 medium to an optical density at $600 \mathrm{~nm}$ of about 0.7. Measures of $\mathrm{OD}_{600 \mathrm{~nm}}$ were performed with the Genesys 20 spectrophotometer (Thermo scientific, Illkirch, France). Cells were diluted until $\mathrm{OD}_{600 \mathrm{~nm}}=0.05$ into $50 \mathrm{~mL}$ of preheated medium $\left(42^{\circ} \mathrm{C}\right)$ and harvested at early $\left(\mathrm{OD}_{600 \mathrm{~nm}}=0.2\right)$, mid exponential growth phase $\left(\mathrm{OD}_{600 \mathrm{~nm}}=0.6\right)$ or stationary phase (after 1.5 hours at $\mathrm{OD}_{600 \mathrm{~nm}}=1.5$ ) with or without $\mathrm{MMC}$ exposure during 2.5 hours at the half of the minimal inhibitory concentration $(\mathrm{MIC} / 2=0.1 \mu \mathrm{g} / \mathrm{mL}$, for all the S. thermophilus strains used in this study) for genomic DNA or RNA extractions. Cultures were centrifuged at 13, 000 g during $15 \mathrm{~min}$ at $42^{\circ} \mathrm{C}$ and cell pellets were stored at $-80^{\circ} \mathrm{C}$.

\section{DNA manipulation}

DNA quantity along the MMC exposure was investigated by colorimetric DNA dosage [36]. Genomic DNA of $S$. thermophilus was extracted as described previously [37]. Plasmid DNA isolation was performed using Genelute Plasmid Miniprep Kit (Sigma-Aldrich, Lyon, France). DNA fragment recovery was performed using the High Pure PCR Product purification kit (Roche, Neuilly-sur-Seine, France). DNA cloning, ligation and

Table 1 Strains and plasmid used in this study.

\begin{tabular}{|c|c|c|}
\hline Strains or plasmids & Relevant phenotype or genotype & Reference \\
\hline \multicolumn{3}{|l|}{ Strains } \\
\hline \multicolumn{3}{|l|}{ S. thermophilus } \\
\hline CNRZ368 & Wild-type strain carrying ICESt1 & INRA-CNRZ \\
\hline CNRZ385 & Wild-type strain carrying ICESt3 & INRA-CNRZ \\
\hline CNRZ368DICEST1 & Wild-type strain cured from its ICESt1 resident element & $\begin{array}{l}\text { X. Bellanger pers. } \\
\text { com. }\end{array}$ \\
\hline $\begin{array}{l}\text { LMG18311 } \\
\text { ICESt3cat }\end{array}$ & $\begin{array}{l}\text { Wild-type strain carrying ICESt3 tagged with the cat gene inserted in the pseudogene } \text { Yorf385J, } \\
\mathrm{Cm}^{r}\end{array}$ & [10] \\
\hline CNRZ368 ICESt3cat & CNRZ368AICESt1 strain carrying ICESt3cat, $\mathrm{Cm}^{\mathrm{r}}$ & This work \\
\hline \multicolumn{3}{|l|}{ E. coli } \\
\hline $\mathrm{DH} 5 \alpha$ & supE44 lacU169 ( $\phi 80$ lacZ M15) hsdR17 endA1 gyrA96 thi-1 relA1 & [33] \\
\hline \multicolumn{3}{|l|}{ Plasmid } \\
\hline pSL1180 & $3,4 \mathrm{~kb}$, replication origin from $\mathrm{pBR} 322, \mathrm{Amp}^{\mathrm{r}}$ & Amersham \\
\hline
\end{tabular}

Abbreviations: $\mathrm{Cm}^{\mathrm{r}}$, chloramphenicol resistance, $\mathrm{Amp}^{\mathrm{r}}$ : ampicillin resistance. 
restriction enzyme digestion were all carried out according to standard procedures [33] or according to specific recommendations of the supplier (New England Biolabs, Evry, France). PCR primers were designed with the PrimerQuest software http://www.idtdna.com/scitools/ applications/primerquest/ and synthesized by Eurogentec (Angers, France) at $100 \mu \mathrm{M}$. PCR and high fidelity PCR were carried out according to the instructions of the ThermoPol PCR kit (New England Biolabs, Evry, France) and of the Triple Master PCR System (Eppendorf, Le Pecq, France), respectively. Sequencing reactions on RACE PCR amplifications were performed by Cogenics (Beckman Coulter genomics, Villepinte, France).

\section{Reverse transcription PCR (RT-PCR)}

Cell pellets were resuspended in $1 \mathrm{~mL}$ of Kirby mix (1\% $\mathrm{w} / \mathrm{v}$ of $\mathrm{N}$-Lauroylsarcosine, $6 \% \mathrm{w} / \mathrm{v}$-aminosalicylic acid sodium salt, $0.1 \mathrm{M}$ Tris $\mathrm{HCl} \mathrm{pH}=8,6 \% \mathrm{v} / \mathrm{v}$ phenol $\mathrm{pH}$ $=8$ ). Then total RNAs were extracted as described previously [38]. The cDNAs were obtained by reverse transcription of $1 \mu \mathrm{g}$ of DNase I-treated (Euromedex, Souffelweyersheim, France) total RNA with M-MLV reverse transcriptase (Invitrogen, Villebon sur Yvette, France) and random hexamer primers (Applied Biosystems, Villebon sur Yvette, France). PCR amplification of gyrA (40 cycles) was performed using gyrAR1 and gyrAR2 primers (see additional file 3: table S1) on retrotranscribed RNA and non retrotranscribed RNA, and used as positive and negative control, respectively. The quality of generated cDNA was controlled by amplifying a 1000-bp fragment by the J/I.f and G/H.r primers (see additional file 3: table S1). Transcriptional mapping was done using primers amplifying less than 1000-bp with a standard PCR program: $30 \mathrm{~s}$ at $95^{\circ} \mathrm{C}$ for denaturation, annealing $30 \mathrm{~s}$ at $50^{\circ} \mathrm{C}$ and extension $1 \mathrm{~min}$ at $72^{\circ} \mathrm{C}$ for 30 cycles. Primers are listed in the additional file 3 , table $\mathrm{S} 1$ in part and available upon request for the rest.

\section{Mapping of 5' extremity of RNA}

5 ' ends of transcripts were mapped by Rapid Amplification of cDNA Ends using the 5'RACE PCR kit (Invitrogen, Villebon sur Yvette, France). PCR products were directly sequenced to determine the 5 ' ends. When they can not be precisely determined by direct sequencing, PCR products were subsequently cloned in pSL1180 (Table 1); 15 and 12 clones were sequenced for ICESt1 and ICESt3 respectively. Primers used are listed in the additional file 3 table $\mathrm{S} 1$.

\section{Quantitative PCR}

Quantitative PCR (qPCR) was performed with 2 fg-200 ng DNA or cDNA, $5 \mu \mathrm{L}$ qPCR Mastermix (Bio-rad, Marnes-la-Coquette, France) and 450 pM primers (see additional file 3: table S1) in $10 \mu \mathrm{L}$ final volume. After activation of the hot start polymerase $\left(30 \mathrm{~s}\right.$ at $\left.98^{\circ} \mathrm{C}\right), 40$ cycles were performed: denaturation $10 \mathrm{~s}$ at $95^{\circ} \mathrm{C}$ and annealing/extension $45 \mathrm{~s}$ at $50^{\circ} \mathrm{C}$ for cDNA or denaturation $30 \mathrm{~s}$ at $95^{\circ} \mathrm{C}$, annealing $30 \mathrm{~s}$ at $50^{\circ} \mathrm{C}$ and extension $1 \mathrm{~min}$ at $72^{\circ} \mathrm{C}$ for gDNA. The melting curve of the PCR product was analyzed with CFX manager software (Biorad, Marnes-la-Coquette, France) to verify PCR specificity. It was acquired each $0.5^{\circ} \mathrm{C}$ for $1 \mathrm{~s}$ by heating the PCR product from $60^{\circ} \mathrm{C}$ to $95^{\circ} \mathrm{C}$. For each run, a standard dilution of the DNA fragment (preliminary obtained by PCR) was used to check the relative efficiency and quality of primers. A negative control (ultrapure water obtained by the Direct8 Milli-Q system, Millipore, Molsheim, France) was included in all assays. Each reaction was performed at least in duplicate. Realtime PCR was carried out on a C1000 Thermocycler coupled by a CFX96 real-time PCR detection system (Bio-Rad, Marnes-la-Coquette, France). Strains depleted for their resident ICE, CNRZ368 ICESt1 (X. Bellanger unpublished data) and CNRZ385 $\triangle \mathrm{ICESt3}$ [21], which have equal amount of $a t t B$ and $f d a$, were used as controls. cDNA quantities of studied genes were normalized to the amount of cDNA of the gyrA gene, whose transcription is considered as constitutive [39]. Similar results were obtained when the $l d h$ gene, encoding the lactate dehydrogenase, was used for normalization [40]. Data are expressed as mean \pm SD. Statistical analysis was performed with Student's E test. A $p$ value $<0.05$ was considered statistically different.

\section{Sequence analysis}

Protein and nucleic acid sequences from the recombination, regulation and conjugation modules of ICESt1 and ICESt3 were compared with sequences from Firmicutes on the NCBI server http://www.ncbi.nlm.nih.gov using BLASTP, BLASTN and/or tBLASTN. Identified sequences are from ICESpn8140 of S. pneumoniae [GenBank:FR671412[22]] and from the partially or completely sequenced genomes of S. parasanguinis F0405 [GenBank:NZ_AEKM00000000] and ATCC15912 [GeneBank:NZ_ADVN00000000], S. australis ATCC700641 [GeneBank:NZ_AEQR00000000] S. infantis ATCC700779 [GeneBank:NZ_AEVD00000000], S. agalactiae ATCC13813 [GenBank:AEQQ01000089], S. dysgalactiae ATCC12394 [GenBank:CP002215], S. downei F0415 [GenBank:NZ_AEKN01000010], Streptococcus sp. 2_1_36FAA [GenBank:NZ_GG704942] and S. gallolyticus UCN34 [GenBank:NC_013798].

\section{Additional material}

Additional file 1: Fig. S1: Determination of transcriptional units of the ICE core region in stationary phase. ICESt1 (A, B) and ICESt3 (C, D). For (A) and (B), location and orientation of ORFs and a truncated IS 
are indicated by arrowed boxes and rectangle, respectively. Above, ORF names beginning with "orf" are abbreviated with the corresponding letter or number. The pattern of the arrowed boxes depicts the putative function and/or relationships of each ORF deduced from functional analyses or from BLAST comparisons. White arrowed boxes correspond to unrelated ORFs of the two elements. Black arrowed box is the chromosomal fda gene. Star represents the putative origin of transfer. Horizontal lines delimitate functional modules with their names above. Arrows below each ICE represent transcripts deduced from the results given in B and D. For (B) and (D), RT-PCR amplification was used to determine if RNA spans the ORF end and the beginning of the following or next ORF. For each amplifications, the positive control performed on genomic DNA is presented on the left and the amplification obtained on CDNA is showed on the right. ORFs named above indicate the examined region and numbers below indicate the calculated amplicon size. Similar results were generated with RNA from three independent biological replicates and cells in exponential growth phase. A PCR was performed without reverse transcriptase step, in order to control for the absence of DNA contamination (not shown).

Additional file 2: Fig. S2: Multiple alignment of the four promoter regions of the seven closely related streptococcal ICEs. (A) $\mathrm{P}_{\text {orfo, }}$ (B) $P_{\text {cri }}$ (C) $P_{\text {arp2 }}$ and (D). Parp2s. Spara_15912, S. parasanguinis ATCC15912; Sinf_700779, S. infantis ATCC 700779; ICESpn8140 from S. pneumoniae 8140; Saus_700641, S. australis ATCC700641; Spara_F0405, S. parasanguinis F0405. The -10 and -35 boxes of the promoters are grey coloured and the transcriptional start sites $(+1)$ are in boldface. For $\mathrm{P}_{\text {orf }}$ region $(A)$, the change in free energy $(\Delta G)$ of the underlined terminator is indicated on the right. For $P_{\text {arp2 }}$ region (C), horizontal lines below the sequences delimitate the putative stems regions and dashed lines the loop parts, which might be involved in mRNA cleavage.

Additional file 3: Table S1. Main primers used in this study.

\section{Acknowledgements}

We thank S. Payot-Lacroix and J.B. Vincourt for critical reading of the manuscript. NC is supported by MNERT fellowship from the Ministère de I'Education et de la Recherche. The authors are grateful to X. Bellanger for CNRZ368 $\triangle$ St1 and M. Mourou for help with the CNRZ368 ICESt3cat.

\section{Author details}

'Nancy-Université, UMR1128, Génétique et Microbiologie, F-54506 Vandœuvre-lès-Nancy, France. ${ }^{2}$ INRA, UMR1128, Génétique et Microbiologie, F-54506 Vandœuvre-lès-Nancy, France.

\section{Authors' contributions}

Conceived and designed the experiments: NC VL FCB PL GG. Performed the experiments: NC VL CM. Analyzed the data: NC VL FCB PL BD GG. Wrote the paper: NC VL FCB GG. All authors read and approved the final manuscript.

Received: 24 June 2011 Accepted: 24 October 2011

Published: 24 October 2011

\section{References}

1. Dobrindt U, Hochhut B, Hentschel U, Hacker J: Genomic islands in pathogenic and environmental microorganisms. Nat Rev Microbiol 2004, 2:414-424

2. Hacker J, Carniel E: Ecological fitness, genomic islands and bacterial pathogenicity. A Darwinian view of the evolution of microbes. EMBO Rep 2001, 2:376-381.

3. Burrus V, Pavlovic G, Decaris B, Guédon G: Conjugative transposons: the tip of the iceberg. Mol Microbiol 2002, 46:601-610.

4. Brochet M, Rusniok C, Couvé E, Dramsi S, Poyart C, Trieu-Cuot P, Kunst F, Glaser P: Shaping a bacterial genome by large chromosomal replacements, the evolutionary history of Streptococcus agalactiae. Proc Natl Acad Sci USA 2008, 105:15961-15966.

5. Wozniak RAF, Waldor MK: Integrative and conjugative elements: mosaic mobile genetic elements enabling dynamic lateral gene flow. Nat Rev Microbiol 2010, 8:552-563.
6. Roberts AP, Johanesen PA, Lyras D, Mullany P, Rood Jl: Comparison of Tn5397 from Clostridium difficile, Tn916 from Enterococcus faecalis and the CW459tet(M) element from Clostridium perfringens shows that they have similar conjugation regions but different insertion and excision modules. Microbiology (Reading, Engl.) 2001, 147:1243-1251.

7. Garnier F, Taourit S, Glaser P, Courvalin P, Galimand M: Characterization of transposon Tn1549, conferring VanB-type resistance in Enterococcus spp. Microbiology (Reading, Engl.) 2000, 146(Pt 6):1481-1489.

8. Burrus V, Pavlovic G, Decaris B, Guédon G: The ICESt1 element of Streptococcus thermophilus belongs to a large family of integrative and conjugative elements that exchange modules and change their specificity of integration. Plasmid 2002, 48:77-97.

9. Pavlovic G, Burrus V, Gintz B, Decaris B, Guédon G: Evolution of genomic islands by deletion and tandem accretion by site-specific recombination: ICESt1-related elements from Streptococcus thermophilus. Microbiology (Reading, Engl.) 2004, 150:759-774.

10. Bellanger X, Roberts AP, Morel C, Choulet F, Pavlovic G, Mullany P, Decaris $B$, Guédon $G$ : Conjugative transfer of the integrative conjugative elements ICESt1 and ICESt3 from Streptococcus thermophilus. J Bacteriol 2009, 191:2764-2775.

11. Bellanger X, Morel C, Decaris B, Guédon G: Derepression of excision of integrative and potentially conjugative elements from Streptococcus thermophilus by DNA damage response: implication of a cl-related repressor. J Bacteriol 2007, 189:1478-1481.

12. Bose B, Auchtung JM, Lee CA, Grossman AD: A conserved anti-repressor controls horizontal gene transfer by proteolysis. Mol Microbiol 2008, 70:570-582.

13. Dodd IB, Shearwin KE, Egan JB: Revisited gene regulation in bacteriophage lambda. Curr Opin Genet Dev 2005, 15:145-152.

14. Beaber JW, Burrus V, Hochhut B, Waldor MK: Comparison of SXT and R391, two conjugative integrating elements: definition of a genetic backbone for the mobilization of resistance determinants. Cell Mol Life Sci 2002, 59:2065-2070.

15. Beaber JW, Hochhut B, Waldor MK: SOS response promotes horizontal dissemination of antibiotic resistance genes. Nature 2004, 427:72-74.

16. Bose B, Grossman AD: Regulation of horizontal gene transfer in Bacillus subtilis by activation of a conserved site-specific protease. J Bacteriol 2011, 193:22-29.

17. Auchtung JM, Lee CA, Monson RE, Lehman AP, Grossman AD: Regulation of a Bacillus subtilis mobile genetic element by intercellular signaling and the global DNA damage response. Proc Natl Acad Sci USA 2005, 102:12554-12559.

18. Ramsay JP, Sullivan JT, Jambari N, Ortori CA, Heeb S, Williams P, Barrett DA, Lamont IL, Ronson CW: A LuxRI-family regulatory system controls excision and transfer of the Mesorhizobium loti strain R7A symbiosis island by activating expression of two conserved hypothetical genes. Mol Microbiol 2009, 73:1141-1155.

19. RNAfold web server. [http://rna.tbi.univie.ac.at/cgi-bin/RNAfold.cgi].

20. Solaiman DK, Somkuti GA: Isolation and characterization of transcription signal sequences from Streptococcus thermophilus. Curr Microbiol 1997, 34:216-219.

21. Bellanger $X$, Morel C, Gonot F, Puymège A, Decaris B, Guédon G: Sitespecific accretion of an Integrative Conjugative Element and a related genomic island leads to cis-mobilization and gene capture. Mol Microbiol 2011, Accepted.

22. Croucher NJ, Harris SR, Fraser C, Quail MA, Burton J, van der Linden $M$, McGee L, von Gottberg A, Song JH, Ko KS, Pichon B, Baker S, Parry CM, Lambertsen LM, Shahinas D, Pillai DR, Mitchell TJ, Dougan G, Tomasz A, Klugman KP, Parkhill J, Hanage WP, Bentley SD: Rapid pneumococcal evolution in response to clinical interventions. Science 2011, 331:430-434

23. Sitkiewicz I, Green NM, Guo N, Mereghetti L, Musser JM: Lateral gene transfer of streptococcal ICE element RD2 (region of difference 2) encoding secreted proteins. BMC Microbiol 2011, 11:65.

24. Goranov Al, Kuester-Schoeck E, Wang JD, Grossman AD: Characterization of the global transcriptional responses to different types of DNA damage and disruption of replication in Bacillus subtilis. J Bacteriol 2006, 188:5595-5605.

25. Auchtung JM, Lee CA, Garrison KL, Grossman AD: Identification and characterization of the immunity repressor (ImmR) that controls the mobile genetic element ICEBs1 of Bacillus subtilis. Mol Microbiol 2007, 64:1515-1528. 
26. Celli J, Trieu-Cuot P: Circularization of Tn916 is required for expression of the transposon-encoded transfer functions: characterization of long tetracycline-inducible transcripts reading through the attachment site. Mol Microbiol 1998, 28:103-117.

27. Lee CA, Babic A, Grossman AD: Autonomous plasmid-like replication of a conjugative transposon. Mol Microbiol 2010, 75:268-279.

28. Klockgether J, Würdemann D, Reva O, Wiehlmann L, Tümmler B: Diversity of the abundant pKLC102/PAGl-2 family of genomic islands in Pseudomonas aeruginosa. J Bacteriol 2007, 189:2443-2459.

29. Doléans-Jordheim A, Akermi M, Ginevra C, Cazalet C, Kay E, Schneider D, Buchrieser C, Atlan D, Vandenesch F, Etienne J, Jarraud S: Growth-phasedependent mobility of the Ivh-encoding region in Legionella pneumophila strain Paris. Microbiology (Reading, Engl.) 2006, 152:3561-3568.

30. Juhas M, Power PM, Harding RM, Ferguson DJP, Dimopoulou ID, Elamin AR e, Mohd-Zain Z, Hood DW, Adegbola R, Erwin A, Smith A, Munson RS, Harrison A, Mansfield L, Bentley S, Crook DW: Sequence and functional analyses of Haemophilus spp. genomic islands. Genome Biol 2007, 8:R237.

31. Mohd-Zain Z, Turner SL, Cerdeño-Tárraga AM, Lilley AK, Inzana TJ, Duncan AJ, Harding RM, Hood DW, Peto TE, Crook DW: Transferable antibiotic resistance elements in Haemophilus influenzae share a common evolutionary origin with a diverse family of syntenic genomic islands. J Bacteriol 2004, 186:8114-8122.

32. McLeod SM, Burrus V, Waldor MK: Requirement for Vibrio cholerae integration host factor in conjugative DNA transfer. J Bacteriol 2006, 188:5704-5711.

33. Sambrook J, David WR: Molecular cloning: a laboratory manual. CSHL Press; 2001.

34. Stingele F, Neeser JR, Mollet B: Identification and characterization of the eps (Exopolysaccharide) gene cluster from Streptococcus thermophilus Sfi6. J Bacteriol 1996, 178:1680-1690.

35. Borges F, Layec S, Fernandez A, Decaris B, Leblond-Bourget N: High genetic variability of the Streptococcus thermophilus cse central part, a repeat rich region required for full cell segregation activity. Antonie Van Leeuwenhoek 2006, 90:245-255.

36. Gerhardt P: Methods for general and molecular bacteriology. Washington D.C.: American Society for Microbiology; 1994.

37. Colmin C, Pebay M, Simonet JM, Decaris B: A species-specific DNA probe obtained from Streptococcus salivarius subsp. thermophilus detects strain restriction polymorphism. FEMS Microbiol Lett 1991, 65:123-128.

38. Kieser T, Bibb MJ, Buttner MJ, Chater KF, Hopwood DA: Practical Streptomyces Genetics. 2e edition. Norwich, England: John Innes Foundation; 2000.

39. Duary RK, Batish VK, Grover S: Expression of the atpD gene in probiotic Lactobacillus plantarum strains under in vitro acidic conditions using RTqPCR. Res Microbiol 2010, 161:399-405.

40. Fernandez A, Thibessard A, Borges F, Gintz B, Decaris B, Leblond-Bourget N: Characterization of oxidative stress-resistant mutants of Streptococcus thermophilus CNRZ368. Arch Microbiol 2004, 182:364-372.

doi:10.1186/1471-2180-11-238

Cite this article as: Carraro et al:: Differential regulation of two closely related integrative and conjugative elements from Streptococcus thermophilus. BMC Microbiology 2011 11:238.

\section{Submit your next manuscript to BioMed Central and take full advantage of:}

- Convenient online submission

- Thorough peer review

- No space constraints or color figure charges

- Immediate publication on acceptance

- Inclusion in PubMed, CAS, Scopus and Google Scholar

- Research which is freely available for redistribution

Submit your manuscript at www.biomedcentral.com/submit
Biomed Central 